\title{
Weight management in overweight and obese breast cancer survivors
}

\begin{abstract}
A large proportion of breast cancer survivors are overweight or obese, putting them at increased risk for chronic diseases, cancer recurrence, and premature mortality. It is therefore necessary to develop weight management interventions that are effective and sustainable. This review discusses pertinent information related to (1) obesity in female breast cancer survivors, and (2) weight management strategies and their effectiveness in overweight and obese female breast cancer survivors. While traditional weight loss interventions in breast cancer survivors combining diet, physical activity, and behavior modification were most effective in achieving weight loss over the short-term, complementary alternative weight loss methods, including mindfulness meditation and mindful eating, appeared to have increased adherence and altered behavior change, including decreased binge eating, emotional eating, food cravings, and sedentary time, over the long-term. More research is needed to examine different types weight management interventions long-term in order to determine their sustainability and effects on morbidity and mortality outcomes over time.
\end{abstract}

Keywords: weight management, obesity, breast cancer, women, nutrition, physical activity, behavior modification, mindfulness, mindful eating
Volume 7 Issue 3 - 2017

\author{
Annette Monique Washington,' Elisabeth \\ Lilian Pia Sattler ${ }^{1,2}$ \\ 'Department of Foods and Nutrition, University of Georgia, \\ USA \\ ${ }^{2}$ Department of Clinical and Administrative Pharmacy, University \\ of Georgia, USA
}

\begin{abstract}
Correspondence: Elisabeth Lilian Pia Sattler, Department of Foods and Nutrition, College of Family and Consumer Sciences, and Department of Clinical and Administrative Pharmacy, College of Pharmacy, University of Georgia, USA, 250W. Green St, Room 270 O R.C. Wilson Pharmacy, Athens, Georgia, 30602, USA, Tel + |7065421040, Fax + 17065425228, Email lilian@uga.edu
\end{abstract}

Received:September 12, 2017 | Published: September 25, 2017
Abbreviations: DXA, dual energy x-ray absorptiometry; SERMs, selective estrogen receptor modulators; BMI, body mass index; ME, mindful eating

\section{Introduction}

It is estimated that 3.1 million women in the United States are breast cancer survivors. ${ }^{1}$ Approximately $50 \%$ of breast cancer survivors are overweight or obese, which increases their risk for chronic disease development and premature mortality. ${ }^{2}$ Many affected women remain weight stable during cancer treatment but report weight gain in the months and years after diagnosis. ${ }^{3}$ Even in the absence of weight gain, there are unfavorable changes in body composition, including gain in fat and loss in lean tissue mass. Excess body weight at or after diagnosis is associated with a $20-43 \%$ higher risk of breast cancer specific and overall mortality, and twice the risk of breast cancer recurrence, compared to healthy weight women. ${ }^{4}$ Moreover, women diagnosed with breast cancer, in comparison to women without breast cancer, are more likely to develop cardiovascular diseases, which are strongly associated with overweight and obesity. ${ }^{5}$ Given the increased morbidity and mortality risk, maintenance of a healthy body weight, or modest weight loss, is strongly recommended in this population. ${ }^{4,6}$ This review summarizes pertinent information related to obesity in female breast cancer survivors, and discusses weight management strategies and their effectiveness in overweight and obese female breast cancer survivors.

\section{Discussion}

\section{Overweight and obesity in breast cancer survivors}

Multiple factors likely play a role in the development and maintenance of obesity in breast cancer survivors. First, systemic treatments, particularly chemotherapy, have been shown to lead to significant weight gain, although the underlying mechanisms are unclear. ${ }^{3}$ Specifically, duration of chemotherapy has been examined as predictor of weight gain. ${ }^{3}$ A prospective study in pre- and postmenopausal women with breast cancer showed that $34 \%$ of breast cancer survivors treated with chemotherapy gained between 2.5$5 \mathrm{~kg}$ weight, as compared to women who were not. ${ }^{3}$ It was also not uncommon to see weight gain of more than $10 \mathrm{~kg}$ during treatment which the authors assumed to be due to a combination of reasons, including but not limited to stress eating, reduced physical activity, lowered metabolic rate from chemotherapy, and the chemotherapy medications themselves. ${ }^{7}$ A review examining weight gain in breast cancer survivors found that women treated with chemotherapy were $65 \%$ more likely to gain weight during treatment than those who received localized treatment (i.e., surgery). ${ }^{3}$ Selective Estrogen Receptor Modulators (SERMs), which are used to treat breast cancer along with chemotherapy, have been shown in earlier studies to lead to weight gain, however, recent and larger studies have shown that SERM treatment did not to lead to any significant weight gain. ${ }^{3,8}$ Length of SERM treatment was also not a significant variable. ${ }^{8}$ While the type of chemotherapy may play a role in weight gain, the duration of treatment may be important as well. Shorter chemotherapy treatments have shown to lead to minimal, non-significant weight gain, as compared to longer treatments, yet these findings are still inconsistent. ${ }^{3,8}$ In addition to chemotherapy, radiation therapy has shown to lead to an increase in body fat percentage and reduction in fat free mass in breast cancer survivors, as measured by Dual-energy X-ray Absorptiometry (DXA). ${ }^{3}$ Radiation therapy was also associated with a $50 \%$ reduction in physical activity levels, as compared to the $24 \%$ reduction in those who were treated by surgery alone. ${ }^{8}$ This has been confirmed by several studies showing that there is a significant reduction in physical activity during and after treatment, which may partially explain weight gain. ${ }^{3}$ It is likely that the detrimental effects of cancer treatments on patient well-being lead to unfavorable lifestyle behaviors, which in turn affect energy balance that may explain weight gain not directly associated with chemotherapy. ${ }^{3}$ 
While evidence-based guidelines for lifestyle management, including nutrition and physical activity, differentiate between cancer survivors in treatment vs. cancer survivors in remission, particularly the latter group struggles with long-term weight management which underlines the importance of weight management interventions tailored towards this population. ${ }^{6}$

\section{Traditional weight management interventions in breast cancer survivors}

Substantial evidence shows a positive effect of weight loss interventions on breast cancer survivors' weight and waist circumference. ${ }^{4}$ A systematic review by Reeves et al., ${ }^{4}$ reported that weight loss interventions were not only safe, but were also successful in achieving weight loss and reductions in central adiposity in breast cancer survivors. In particular, interventions that combined diet, physical activity, and behavior modification were most effective in achieving a weight loss of $\geq 5 \%$ of body weight which reflects the recommendation for comorbidity management in this population. ${ }^{4}$ Rock et al., ${ }^{9}$ conducted a 2-year, cognitive-behavioral weight loss randomized controlled trial of 692 obese postmenopausal breast cancer survivors. The intensive weight loss intervention took place during the first year, and included weekly, group-based, nutrition and physical activity sessions, telephone counseling, and tailored newsletters. After 1-year study duration, $55 \%$ of the participants had lost $\geq 5 \%$ of their baseline body weight. During the second year, the telephone counseling and weekly sessions were significantly reduced. By the end of year 2, only $44 \%$ of the intervention group participants had lost $\geq 5 \%$ of their baseline body weight. ${ }^{9}$ The study showed how intensive weight loss programs can lead to prevention of weight regain in this population. ${ }^{9}$ Further, in a 12-week single-arm intervention study, 37 overweight breast cancer survivors completed a diet and physical activity intervention. ${ }^{10}$ The intervention included weekly meetings with a dietitian, and biweekly exercise sessions with trained physical activity monitors. ${ }^{10}$ After the intervention, an average of $7.8 \pm 2.9 \%$ decrease in the participants' baseline weight was observed, along with significant positive changes in Body Mass Index (BMI), waist and hip circumference, fat percentage and fat mass. ${ }^{10}$ In an 8-week weight loss intervention study, breast cancer survivors met with an exercise physiologist three times a week, and a nutritionist at baseline and multiple times via telephone. ${ }^{11}$ At follow-up, there was a significant decrease in weight, body fat percentage, and waist and hip circumference, and a significant increase in lean body mass. ${ }^{11}$ These results were confirmed by other studies that have shown significant weight loss in the target population in interventions lasting less than 6months. ${ }^{10}$

\section{Mindfulness, mindful eating, and obesity outcomes in breast cancer survivors}

Breast cancer survivors are increasingly exploring complementary alternative options after treatment, including mindfulness-based meditation and mindful eating (ME), to help alleviate the psychological and emotional stress that is gained during cancer diagnosis and treatment. ${ }^{12}$ In addition, mindfulness-based studies have reported positive effects on body weight, including weight loss and weight maintenance in overweight and obese individuals. ${ }^{13}$ Mindfulness is defined as a non-judgmental awareness of the present moment. ${ }^{13}$ It is associated with the calming of emotions and self-regulation and an overall increase in physical activity levels. ${ }^{12}$ Mindfulness may indirectly address the post-diagnosis components of weight gain in breast cancer survivors, including sedentariness and dietary intake. ${ }^{12}$ Mindful eating is a branch of mindfulness meditation that includes consuming food with intention and attention, and identifying emotional triggers that cause overconsumption. ${ }^{14}$ Traditional diet restriction interventions have shown only short-term success in weight management, with weight regain typically occurring months into the program. ${ }^{7}$ Mindful eating has been suggested as a complementary alternative weight loss strategy due to better long-term sustainability. ${ }^{14}$ In addition, ME has been associated with reducing behaviors associated with weight gain, such as binge eating, emotional eating, and food cravings. ${ }^{15}$ According to a large-scale, prospective cohort study in a sample of women representative of the general population, women with higher mindfulness scores were more likely to have a lower BMI and were more likely to be physically active than women with lower scores. ${ }^{13}$ Inversely, women with lower mindfulness scores were more likely to be struggling with unhealthy eating behaviors, such as emotional eating, overconsumption, binge eating, and food cravings. ${ }^{13}$ In addition, a systematic review and meta-analysis noted that mindful/ attentive-eating as an intervention increased the participants' memory of the food that was consumed, thereby reducing their food intake a later occasion. ${ }^{14}$ It was also noted that lower awareness led to increased immediate food intake. ${ }^{14}$ It was suggested that this method be used in place of conscious calorie counting because patients were more mindful of total daily energy consumption in the long-term. ${ }^{14}$ Although the effects of mindfulness and ME as complementary alternative weight loss strategies have not been well studied in breast cancer survivors, there is promising evidence at the horizon. Larkey et al., ${ }^{12}$ proposed a biobehavioral model supporting mindfulness meditation and weight loss in breast cancer survivors. The authors suggested that mindfulness meditation would lead to physiological, psychological, and biological responses that would directly and indirectly address weight gain in this population. While mindfulness may not inherently lead to energy expenditure, this model was based on literature showing that mindfulness was associated with increased physical activity and decreased sedentary time. ${ }^{12}$ Their conclusion was that sedentariness, dietary intake, and stress would likely be affected long-term, thus again, making mindfulness a sustainable treatment option. ${ }^{12}$ Finally, a single-group, longitudinal study by Chung et al., ${ }^{16}$ examined ME for weight loss in African American breast cancer survivors. At the completion of the 12-week study, there was an increase in mindfulness, and a decrease in weight. There was a significant association between mindful eating scores and weight loss amongst this cohort. ${ }^{16}$

\section{Conclusion}

Traditional weight loss interventions in breast cancer survivors combining diet, physical activity, and behavior modification have shown effectiveness in achieving weight loss over the short-term. There is growing evidence that complementary alternative weight loss methods, including mindfulness meditation and ME have potential to alter unfavorable nutritional intake behaviors, including decreased binge eating, emotional eating, food cravings, and sedentary time over the long-term. More research is needed to examine different types weight management interventions long-term in order to determine their sustainability and effects on morbidity and mortality outcomes in breast cancer survivors over time.

\section{Acknowledgements}

This work was partly supported by the University of Georgia Clinical and Translational Research Unit Grant. 


\section{Conflict of interest}

No financial interests or conflicts of interest exist for any of the contributing authors.

\section{References}

1. Breast Cancer Facts \& Figures 2015-2016. USA: American Cancer Society Inc.

2. Thompson HJ, Sedlacek SM, Playdon MC, et al. Weight loss interventions for breast cancer survivors: impact of dietary pattern. PloS one. $2015 ; 10(5): \mathrm{e} 0127366$

3. Vance V, Mourtzakis M, McCargar L, et al. Weight gain in breast cancer survivors: prevalence, pattern and health consequences. Obesity reviews. 2011;12(4):282-294.

4. Reeves MM, Terranova CO, Eakin EG, et al. Weight loss intervention trials in women with breast cancer: a systematic review. Obes rev. 2014;15(9):749-768.

5. Bardia A, Arieas ET, Zhang Z, et al. Comparison of breast cancer recurrence risk and cardiovascular disease incidence risk among postmenopausal women with breast cancer. Breast Canc Res Treat. 2012;131(3):907-914.

6. Rock CL, Doyle C, Demark-Wahnefried W, et al. Nutrition and physical activity guidelines for cancer survivors. $C A$ : A Canc J Clin 2012;62(4):242-274

7. Hamer J, Warner E. Lifestyle modifications for patients with breast cancer to improve prognosis and optimize overall health. Canadian Medical Association Journal. 2017;189(7):268E -274E.

8. Makari-Judson G, Braun B, Jerry DJ, et al. Weight gain following breast cancer diagnosis: implication and proposed mechanisms. World J Clin Oncol. 2014;5(3):272-282.
9. Rock CL, Flatt SW, Byers TE, et al. Results of the exercise and nutrition to enhance recovery and good health for you (ENERGY) trial: a behavioral weight loss intervention in overweight or obese breast cancer survivors. J Clin Oncol. 2015;33(28):3169-3176.

10. Travier N, Fonseca Nunes A, Javierre C, et al. Effect of a diet and physical activity intervention on body weight and nutritional patterns in overweight and obese breast cancer survivors. Med Oncol. 2014;31(1):783-785.

11. Mc Tiernan A, Ulrich C, Kumai C, et al. Anthropometric and hormone effects of an eight-week exercise-diet intervention in breast cancer patients: results of a pilot study. Cancer epidemiology, biomarkers \& prevention. 1998;7(6):477-481.

12. Larkey LK, Vega-López S, Keller C, et al. A biobehavioral model of weight loss associated with meditative movement practice among breast cancer survivors. Health Psychology Open. 2014;1(1):1.

13. Camilleri GM, Mejean C, Bellisle F, et al. Association between mindfulness and weight status in a general population from the NutrinetSante study. PloS one. 2015;10(6):e0127447.

14. Robinson E, Aveyard P, Daley A, et al. Eating attentively: a systematic review and meta-analysis of the effect of food intake memory and awareness on eating. Amer J Clin Nutr. 2013;97(4):728-742.

15. O'Reilly GA, Cook L, Spruijt-Metz D, et al. Mindfulness-based interventions for obesity-related eating behaviours: a literature review. Obes Rev. 2014;15(6):453-461.

16. Chung S, Zhu S, Friedmann E, et al. Weight loss with mindful eating in African American women following treatment for breast cancer: a longitudinal study. Support Care Cancer. 2016;24(4):1875-1881. 